\title{
Investigação da lateralidade em termos de preferência e dominância manual em primatas Sapajus apella
}

\section{Investigation of laterality in terms of hand preference and hand dominance in Sapajus apella primates}

Leon Claudio Pinheiro Leal ${ }^{1,2}$ (D), Rafaela Martins Reis ${ }^{1,2}$ iD, José Augusto Pereira Carneiro Muniz ${ }^{2}$, Carlomagno Pacheco Bahia ${ }^{1}$, Lane Viana Krejcovál, 2 iD

1 Universidade Federal do Pará, Instituto de Ciências da Saúde, Laboratório de Neuroplasticidade, Belém, Pará, Brasil

2 Instituto Evandro Chagas/SVS/MS, Centro Nacional de Primatas, Ananindeua, Pará, Brasil

\section{RESUMO}

OBJETIVO: Quantificar a variabilidade individual na lateralidade de primatas Sapajus apella, a fim de determinar a ocorrência de preferência e/ou dominância manual por meio de dois testes motores. MATERIAIS E MÉTODOS: Sete macacos machos adultos foram submetidos a sessões de habituação aos experimentadores e aparatos experimentais, seguidos de 20 sessões dos testes motores staircase e tabuleiro de Brinkman, que permitem medir as habilidades de alcance e pegada e a precisão dos movimentos em pinça. Parâmetros, como latência de retirada da primeira recompensa, uso de mão trocada, tempo para a retirada de todas as recompensas e perda da recompensa, foram coletados para cada mão individualmente. Análises da sequência de retirada das recompensas no tabuleiro de Brinkman foram realizadas para observação de estabelecimento de padrões motores. RESULTADOS: Foram observadas diferenças significativas entre os dados obtidos para as mãos direita e esquerda para todos os animais, com variações importantes na apresentação de preferência e dominância manuais, bem como alterações no padrão motor de realização do teste com indicativos de aprendizado induzido pelo mesmo. CONCLUSÃO: Os macacos apresentaram preferência manual na realização de tarefas motoras, com maior prevalência de preferência pelo lado direito, não havendo necessária correspondência entre preferência e dominância manual para cada animal.

Palavras-chave: Primatas; Lateralidade; Preferência Manual; Dominância Manual.

\begin{abstract}
OBJECTIVE: To quantify individual variability in the laterality of Sapajus apella primates to determine the occurrence of hand preference and hand dominance, or both, through two motor tests. MATERIALS AND METHODS: Seven adult male monkeys were subjected to sessions of habituation to the experimenters and experimental apparatus, followed by 20 sessions of the staircase and Brinkman board motor tests, which allow the measurement of reach and grip skills, and the precision in the pincer grasp. Parameters such as withdrawal latency for the first reward, usage of switched hand, time to withdraw all rewards, and loss of the reward were collected for each hand individually. Sequence analysis of reward withdrawal on the Brinkman board was performed to observe the establishment of motor patterns. RESULTS: Significant differences were observed between the data obtained for the right and left hands for all animals, with important variations in manual preference and manual dominance, as well as changes in the motor pattern while performing the test with indications of learning induced by it. CONCLUSION: Monkeys showed a hand preference in performing motor tasks, with a higher prevalence of preference on the right side, with no correspondence between hand preference and hand dominance for each animal.
\end{abstract}

Keywords: Primates; Laterality; Manual Preference; Manual Dominance.

\footnotetext{
Correspondência / Correspondence:

Lane Viana Krejcová

Universidade Federal do Pará, Instituto de Ciências da Saúde

Av. Generalíssimo Deodoro, 01. Bairro: Umarizal. CEP: 66050-160 - Belém, Pará, Brasil - Tel.: +55 (91) 98899-5477

E-mail: lanekrejcova@gmail.com
} 


\section{INTRODUÇÃO}

Do ponto de vista evolutivo, o desenvolvimento de destreza manual (habilidade de controlar precisa e independentemente $\mathrm{O}$ movimento de cada um dos dedos) é uma prerrogativa dos primatas, não sendo exibida em grau elevado por nenhuma outra ordem entre os mamíferos. Tal habilidade baseia-se nas especificidades dos circuitos neurais do sistema motor dos primatas, incluindo o trato corticoespinhal bem desenvolvido', que representa uma das muitas similaridades funcionais e estruturais entre o cérebro dos primatas não humanos (doravante denominados primatas) e o cérebro humano, juntamente com assimetrias cerebrais, que em humanos determinam os fenômenos de dominância inter-hemisférica e lateralização de funções.

Diversos estudos analisaram aspectos comportamentais e fisiológicos envolvidos na determinação da lateralização e destreza manual em primatas $^{2,3,4}$, com hipóteses sendo levantadas acerca da possível relação com a dominância hemisférica e destreza observada em humanos. Todavia, a maior parte dos estudos dedicados à investigação dos fenômenos de lateralidade em primatas comumente os determinam por meio da observação da preferência manual, sem enfatizar a distinção entre dois atributos importantes relacionados à lateralização: a preferência e a dominância manual.

Os termos "dominância manual" e "preferência manual" referem-se ao uso consistente de uma mão ao invés de outra para a realização de tarefas que requerem habilidade e coordenação motora, e estão relacionados à lateralização funcional inter-hemisférica ${ }^{5}$. Apesar de serem termos comumente utilizados como sinônimos, existem diferenças essenciais entre os atributos de dominância e preferência manual, bem como na sua apresentação em primatas humanos e não humanos ${ }^{6}$. A preferência manual pode ser definida como a mão escolhida prioritariamente para a realização de uma determinada tarefa, independentemente do fato de esta ser ou não a mais eficiente para a tarefa. A dominância manual, por sua vez, reflete o melhor desempenho e eficiência motora de uma mão em relação à outra em testes que requeiram destreza ${ }^{7}$.

Em humanos, assume-se comumente uma interdependência entre os atributos de preferência e dominância manual, devido ao iá bem estabelecido fenômeno de dominância hemisférica e suas relações com o desenvolvimento cognitivo ${ }^{8}$. Apesar de tal interdependência ser defendida pela geração de um ciclo de retroalimentação positiva, no qual a mão com maior destreza é preferencialmente utilizada, reforçando seu desempenho motor pelo uso constante, há indícios de que dominância e preferência não correspondem de forma sistemática. Evidências demonstram o aparecimento da preferência manual desde o período pré-natal em humanos", bem como a não necessária correspondência entre preferência e dominância manuais para primatas humanos e não humanos ${ }^{6}$.

A ocorrência de preferência manual tem sido demonstrada em diversas espécies de primatas. Porém, de forma distinta ao caso humano, onde cerca de $90 \%$ da população é destra ${ }^{10}$, as porcentagens podem variar grandemente entre primatas ${ }^{11}$, de acordo com a espécie e a população ${ }^{12}$, sendo que a direção da lateralização pode ser acentuada pela idade ou pelo treinamento de tarefas motoras ${ }^{4}$. Não obstante, em diversas espécies de primatas, de forma similar ao caso humano, observa-se acentuada preferência pela mão direita a nível populacional $13,14,15,16,17,18,19$. Em primatas do gênero Cebus (atualmente Sapajus ${ }^{20,21, *}$ ), estudos confirmaram tal predominância, demonstrando seu estabelecimento desde o início da vida ${ }^{22,23,24}$ e sua correlação com assimetrias estruturais no córtex motor primário, em similitude com o caso humano ${ }^{25}$, o que sugere que macacos Cebus consistem em modelos apropriados para estudos da evolução da lateralidade em humanos.

Todavia, apesar da preferência manual já haver sido demonstrada em diversas espécies de primatas, incluindo prossímios, grandes macacos africanos e primatas do gênero Cebus ${ }^{13-19,22,23,25,26}$, não existem evidências irrefutáveis acerca da apresentação do fenômeno de dominância manual entre primatas, sendo questionável a possível homologia dos fenômenos de destreza e lateralidade entre primatas e humanos ${ }^{5}$, apesar de argumentos acerca da influência da complexidade dos testes utilizados sobre os resultados ${ }^{6,17,26}$.

Fatores ambientais, culturais e de aprendizagem podem estar envolvidos na apresentação dos atributos de lateralização, como o aprendizado pela repetição de um teste motor que pode afetar os resultados de testes de lateralidade em primatas ${ }^{6,16}$. Humanos, por exemplo, possuem um amplo repertório motor aprendido, especialmente considerando habilidades bimanuais, nas quais a mão de preferência executa o componente mais complexo da tarefa, enquanto a outra mão desempenha papel de estabilização. Hábitos complexos, como enfiar a linha no buraco de uma agulha, tendem a favorecer a mão com melhor performance motora (dominante), o que induz a coerência entre preferência e dominância em humanos. Em primatas não humanos, as tarefas bimanuais naturais consistem em tarefas motoras simples (ex. descascar uma fruta ou quebrar uma noz), que não representam grandes desafios à coordenação motora fina e podem justificar a maior discrepância entre preferência e dominância manuais observadas nessas espécies. Existem teorias discordantes acerca da homologia ou não da lateralização cerebral entre

\footnotetext{
* Cebus apella, de acordo com a classificação de Hill, incluía todas as espécies de macacos-prego, sendo a espécie amazônica anteriormente conhecida como Cebus apella apella. Todavia, com a reformulação recente da taxonomia das espécies pertencentes ao gênero Cebus, considerando os trabalhos de Silva Junior (2001)20 e Alfaro et al. $(2012)^{21}$, atualmente considera-se apenas uma única espécie de macacos-prego robustos de tufos para a Região Amazônica, os macacos Sapajus apella, conhecidos como macacos-prego, pertencentes à infraordem Platyrrhini, família Cebidae e subfamília Cebinae.
} 
humanos e primatas bem como da influência de fatores ambientais, organização cortical e influências genéticas sobre tal fenômeno $3,8,9,11,19,27,28$, havendo a necessidade de investigações mais profundas nesse cenário.

Ainda, o estudo dos atributos de dominância e preferência manual em primatas, para além de sua importância na observação das similaridades comportamentais e fisiológicas das espécies com os humanos, é essencial e possui implicações relevantes em estudos envolvendo desordens motoras, especialmente unilaterais $^{29,30}$. A cuidadosa avaliação da preferência e dominância manual nesses estudos pode permitir, para além da determinação de sítios de lesão, a obtenção de linhas de base para avaliação da extensão funcional dos danos com base no desempenho individual do animal, e a avaliação precisa dos efeitos de intervenções clínicas, farmacológicas ou cirúrgicas ${ }^{31,32}$, permitindo maior clareza e confiabilidade na interpretação dos dados e adicionando relevância aos dados em cenário pré-clínico.

$O$ presente trabalho tem como objetivo quantificar a variabilidade individual na lateralidade e destreza a fim de determinar a ocorrência dos fenômenos de preferência e dominância manual em macacos $S$. apella, utilizando os testes motores staircase e tabuleiro de Brinkman. A hipótese a ser investigada é a de que diversos parâmetros observáveis nos referidos testes podem predizer a preferência e/ou dominância manual.

\section{MATERIAIS E MÉTODOS}

\section{ANIMAIS}

Foram utilizados sete primatas Sapajus apella machos, normonutridos, provenientes da colônia de reprodução do Centro Nacional de Primatas (CENP) em Ananindeua, estado do Pará. As idades e pesos dos animais estão apresentados no quadro 1.

\begin{tabular}{|ccc|}
\hline Animal & Idade (anos) & Peso $(\mathrm{kg})$ \\
\hline AM-BEM & 16 & 4,200 \\
AM-BEG & 16 & 4,028 \\
AM-AOR & 26 & 3,914 \\
AM-ASA & 22 & 4,538 \\
AM-BBK & 22 & 3,968 \\
AM-BAK & 17 & 3,334 \\
AM-BEK & 16 & 4,800 \\
\hline
\end{tabular}

Quadro 1 - Dados dos animais experimentais

Antes dos experimentos, os indivíduos foram mantidos em grupos de reprodução, formados por dois machos e dez fêmeas, e alocados em gaiolas duplas de 2,5 x 2,0 x 2,5 m cada, com janela de comunicação a qual permitia a livre movimentação dos animais em um espaço de 2,5 x 4,0 × 2,5 m. Os indivíduos selecionados não pertenciam ao mesmo grupo de reprodução e não participaram de outros experimentos. Para a realização dos experimentos, os indivíduos foram realocados em gaiolas duplas individuais contendo um compartimento de $75 \times 75 \times 75 \mathrm{~cm}$ na parte superior e um de mesmas dimensões na parte inferior. $\bigcirc$ acesso entre os compartimentos era feito através de um tubo de passagem de aço inoxidável, permitindo ao animal transitar entre os compartimentos. $\bigcirc$ compartimento inferior continha brinquedos, cordas e aparatos de enriquecimento ambiental que eram trocados diariamente, e o enriquecimento de dieta foi realizado durante todo o período de experimento. As gaiolas com os indivíduos ficaram alocadas lado a lado, em um mesmo galpão, durante todo o período de duração dos experimentos. $\bigcirc$ galpão possuía amplas janelas que proporcionavam iluminação natural, com ciclo claro-escuro regular $(12 / 12 \mathrm{~h})$ durante todo o ano (latitude 01²1'56"S; longitude 48²2'20"W). Os animais foram submetidos a uma dieta constituída por ração própria para animais de laboratório, ofertada diariamente, associada à oferta de frutas e sucos intercalados quatro vezes por semana e água ad libitum. Todos os procedimentos foram realizados de acordo com as diretivas da União Europeia (2010/63/EC) e sob a aprovação da Comissão de Ética para o Uso de Animais do Instituto Evandro Chagas (CEUA/IEC) certificados n. ${ }^{\circ}$ 45/2016 e n. ${ }^{\circ} 37 / 2018$.

\section{PROCEDIMENTOS EXPERIMENTAIS}

\section{Habituação ao experimentador}

Antes do início dos testes motores, os animais foram submetidos a dois meses de habituação com os experimentadores, utilizando a técnica de reforçamento positivo com uso do clicker. Foram realizadas cinco sessões por semana com cada animal. As sessões iniciavam com a retirada do tubo de comunicação e consequente isolamento do animal no compartimento superior da gaiola. A seguir, dois experimentadores (sempre os mesmos, do início ao final do período de experimentos) interagiam de forma ativa com os animais através de oralizações, gestos e toques. A cada interação positiva do animal com os experimentadores (aproximação, oferecimento de partes do corpo para toque e aceitação de recompensas comestíveis com as mãos ou a boca), o experimentador emitia o som com o clicker seguido da oferta de uma pequena recompensa comestível para o animal. As recompensas consistiam em cereais, frutas secas e bolachas, sendo trocadas a cada dia, e aquelas a que cada animal demonstrasse preferência eram listadas para serem utilizadas na realização dos testes motores posteriormente. Todas as recompensas foram avaliadas e liberadas pelo médico veterinário responsável. As sessões de habituação duravam $2 \mathrm{~h}$ cada e eram realizadas antes da primeira oferta da alimentação do dia ao animal; ao término de cada sessão, o tubo de passagem era restituído à gaiola.

\section{Testes motores}

Após o período de habituação, foram realizadas 20 sessões de testes motores, que envolveram o uso de coordenação motora fina e global, para a coleta de dados. Optou-se pela aplicação de um protocolo 
de curta duração para determinar a lateralidade dos animais, de modo a observar o padrão motor natural dos animais, não influenciado pelo aprendizado motor, e consequente aumento de performance de coordenação motora induzida pelos testes ${ }^{27}$. Todavia, análises foram realizadas para verificar a alteração de padrões motores durante o período de realização do teste de Brinkman. Todos os testes utilizaram exclusivamente os membros superiores e foram testados bilateralmente. Foram realizadas cinco sessões por semana, por um período de quatro semanas. A duração de cada sessão variava de acordo com o tempo que o animal levava para o cumprimento de todas as tarefas. Todos os testes foram realizados no período da manhã, uma sessão por dia, antes da primeira alimentação diária, e pelos mesmos dois experimentadores que interagiram com os animais durante as sessões de habituação. Todos os testes foram realizados na mesma sala, na presença dos outros animais. Os testes utilizados estão descritos a seguir.

\section{Teste staircase}

O teste staircase permite avaliar a coordenação motora ampla do membro superior juntamente com a coordenação motora fina do ato da pegada. Foi desenvolvido inicialmente para a avaliação do uso independente das patas dianteiras e habilidade em alcançar e agarrar de ratos e camundongos, sendo posteriormente adaptado para o uso em primatas $^{33,34}$.
Consiste em um aparato de acrílico transparente com duas escadas laterais contendo recompensas comestíveis em seus degraus, de modo que cada escala seja alcançada pelo membro superior de um lado. Os degraus garantem a avaliação da coordenação dos movimentos de agarrar juntamente com a coordenação dos grupamentos adutores, abdutores, flexores e extensores no membro superior durante o movimento de pegada em diferentes níveis do espaço.

Para permitir ests avaliação em diferentes graus de extensão, foram adaptadas duas versões do teste para primatas: valley e hill, que se diferenciam de acordo com o sentido dos degraus em relação ao membro que executa a tarefa. Na tarefa hill, as escadas sobem em direção ao centro, e os orifícios de acesso são posicionados lateralmente, de modo que o animal deve alcançar os degraus do lado esquerdo com o membro superior esquerdo e os degraus do lado direito com o membro superior direito. Em se tratando da valley, as escadas sobem em direção às laterais, e o orifício de acesso localiza-se no centro do aparato, de modo que o animal alcance os degraus do lado direito com o membro superior esquerdo e vice-versa. Para o presente trabalho, foram desenvolvidos aparatos staircase adaptados em suas dimensões para a espécie Sapajus apella a partir das médias do comprimento dos membros superiores de cinco animais, tendo como referência o modelo utilizado por Marshall e Ridley ${ }^{33}$ (Figura 1).
VALLEY
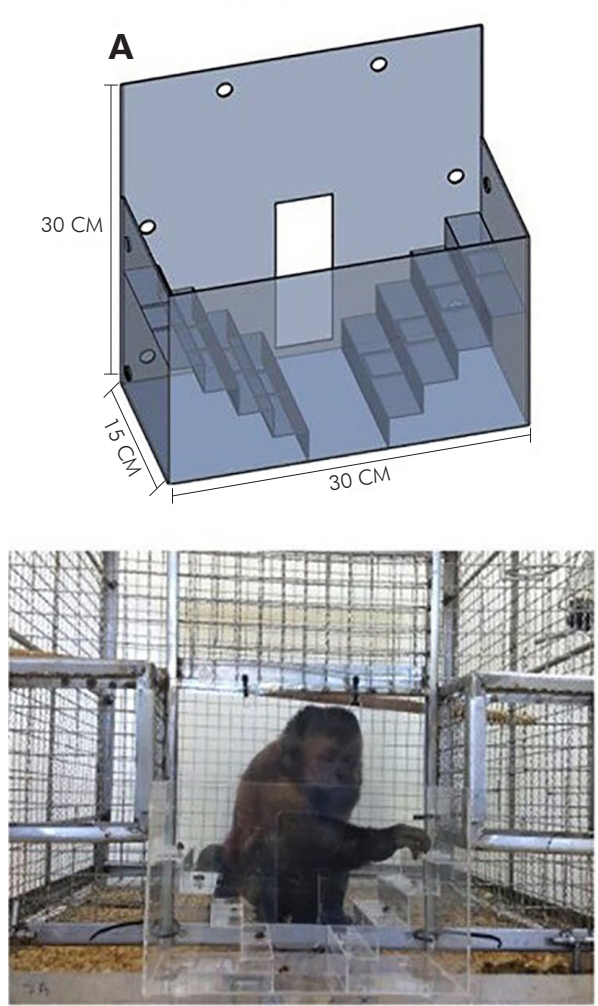

HILL
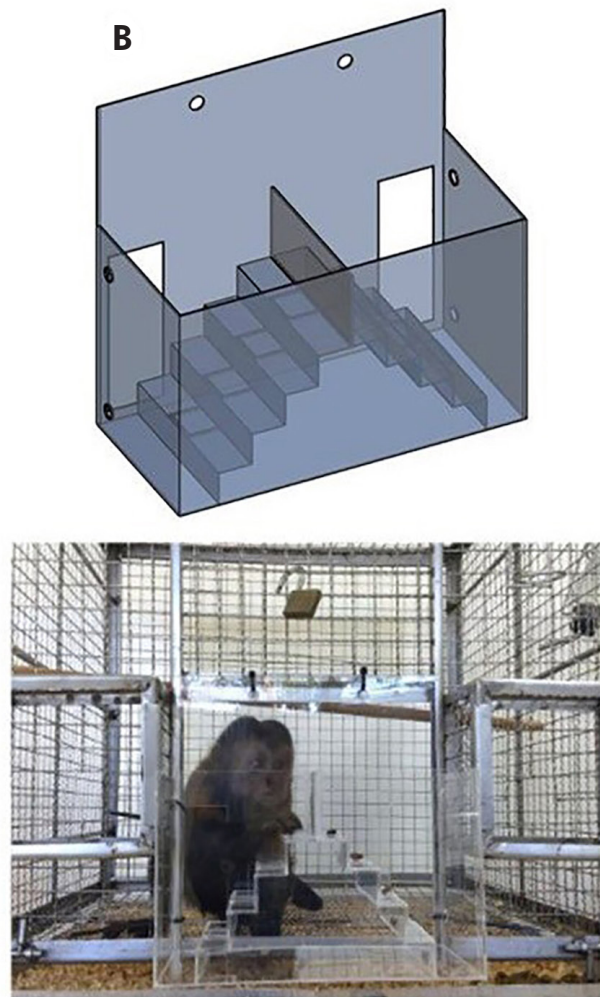

Fotos e ilustrações: Leon Claudio Pinheiro Leal.

A: Modalidade valley, com escadas descendentes à porção medial e orifício de acesso localizado na porção medial do aparato; B: Modalidade hill, com escadas ascendentes à porção medial e orifícios de acesso nas porções laterais direita e esquerda do aparato.

Figura 1 - Aparato experimental para teste staircase 
Antes do início do teste, retirava-se o tubo de passagem isolando o animal na gaiola superior. $\bigcirc$ aparato de teste era posicionado na porta de entrada da gaiola, sendo então fechadas as portinhas de acesso do aparato para colocação das recompensas nos degraus. $O$ teste iniciava com a abertura da portinha de acesso do aparato. Nenhum estímulo para o animal retirar a recompensa foi empregado para além da própria recompensa. $\bigcirc$ teste terminava com a recuperação de todas as recompensas ou após decorrido o tempo máximo de $3 \mathrm{~min}$. As sessões sempre começavam com três tentativas da tarefa hill, seguidas por três tentativas da tarefa valley.

\section{Teste modificado do tabuleiro de Brinkman}

tabuleiro de Brinkman consiste em 50 aberturas retangulares com cantos arredondados, sendo 25 dispostos em posição vertical e 25 em posição horizontal (Figura 2), e é um teste altamente intuitivo que aproveita a habilidade motora e o comportamento natural dos primatas, de forma que macacos não treinados podem facilmente realizá-lo e imediatamente iniciam a retirada das recompensas comestíveis dos compartimentos do tabuleiro quando colocados frente ao mesmo. Ele pode ser realizado de duas formas: simplesmente colocado na frente do animal para a retirada das recompensas, o que revela, a priori, a preferência manual do animal; ou em posição de isolamento de um dos membros, o que foi o caso adotado no presente estudo, uma vez que o objetivo com a aplicação do teste era verificar a possível presença de dominância manual, já que o tabuleiro exige desempenho de coordenação motora fina do ato motor complexo de pegada em pinça, em posições vertical e horizontal ${ }^{35}$. As aberturas eram pequenas o suficiente para permitir somente a inserção de um dedo para retirada da recompensa, de modo que para a retirada da recompensa fosse necessário o uso de pegada em pinça (polegar em oposição ao indicador), o movimento manual de maior precisão entre primatas.

Antes do início do teste, o tubo de passagem era retirado, isolando o animal no compartimento superior da gaiola. Na porta de entrada da mesma, um aparato (cadeira para primatas) era então instalado, permitindo o acesso do animal ao tabuleiro posicionado em uma mesa de madeira, a $45^{\circ}$ do lado a ser testado. $\bigcirc$ aparato possuía aberturas para a passagem dos membros superiores, que eram abertas a cada sessão com o posicionamento ajustado para - lado testado. As recompensas eram colocadas nas aberturas do tabuleiro, e o teste iniciava com a abertura da porta de entrada da gaiola. A conclusão do teste se dava com a recuperação de todas as recompensas do tabuleiro, sem limite máximo de tempo de duração. Durante o teste, não havia qualquer tipo de interferência do avaliador e, ao final, os mesmos procedimentos eram retomados iniciando a realização do teste para o outro lado. A ordem de início do lado a ser testado era alternada para cada sessão.

\section{ANÁLISE DOS DADOS}

\section{Análise de parâmetros do teste staircase}

Todas as sessões de teste foram gravadas e posteriormente analisadas para as seguintes variáveis:

- Latência de retirada da primeira recompensa: tempo que o animal leva para retirar a primeira recompensa com a mão direita ou esquerda, a partir do início da tentativa. É esperado que o animal apresente menor latência para o lado de sua preferência manual, ou seja, inicie a retirada das recompensas com a mão de preferência.

A

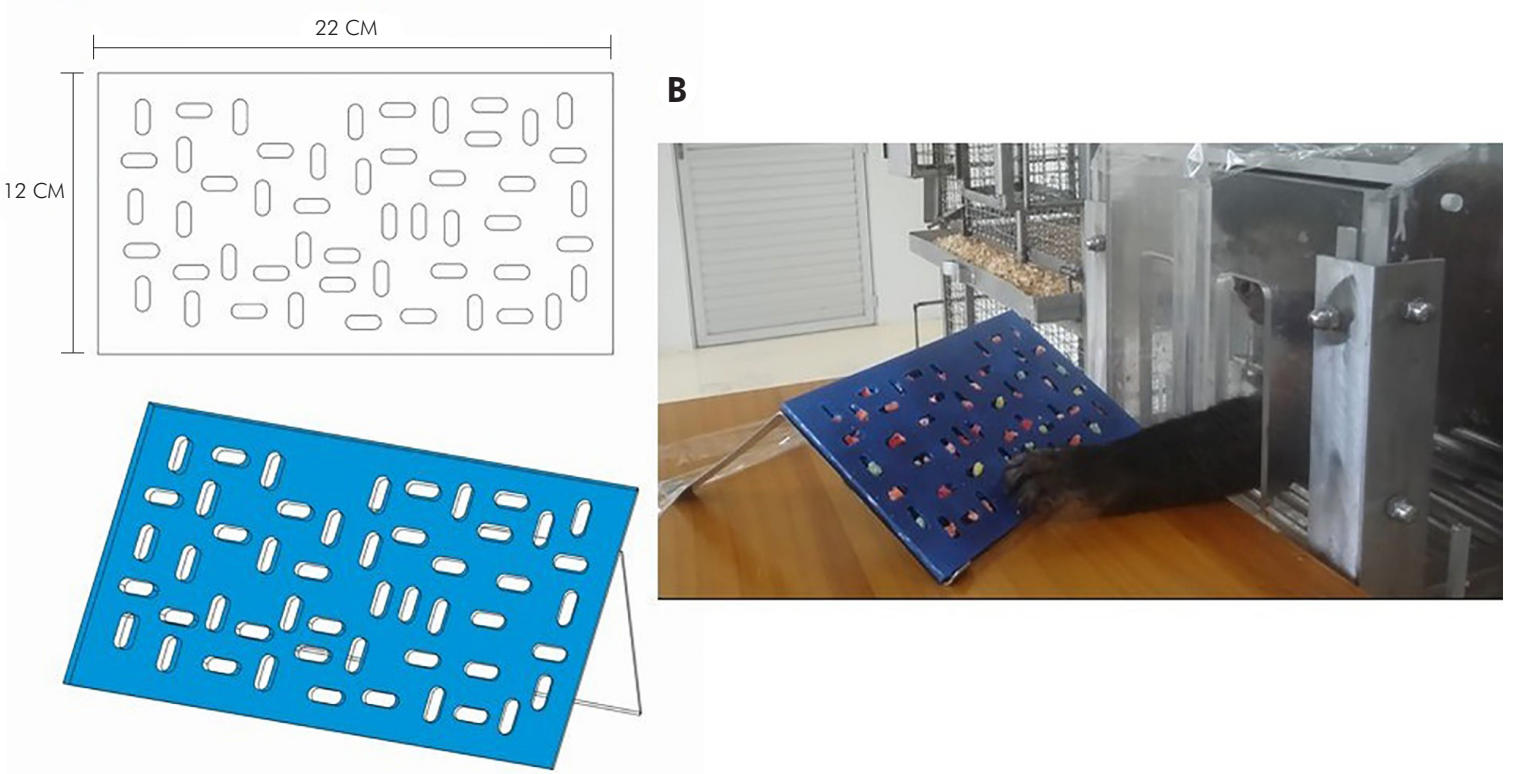

Fotos e ilustrações: Leon Claudio Pinheiro Leal

A: Modelo e dimensões do tabuleiro de Brinkman. B: Posicionamento do tabuleiro em relação à cadeira de primatas, favorecendo a realização do teste para um determinado lado.

Figura 2 - Aparato experimental para teste modificado do tabuleiro de Brinkman 
- Tempo para recuperação de todas as recompensas: tempo total que o animal leva para recuperar todas as recompensas de um determinado lado do aparato; registrado para cada lado separadamente. Nesse parâmetro, o lado dominante deverá apresentar tempos menores para a conclusão da tarefa.

- Uso de mão trocada: número de vezes que o animal tenta fazer a retirada de recompensas de um lado do aparato utilizando a mão contrária à que deve ser utilizada para o referido lado; registrado para ambos os lados. $\bigcirc$ parâmetro revela preferência manual, com maior número de tentativas de realização de ambos os compartimentos do teste utilizando a mão de preferência.

- Perda da recompensa: número de vezes que o animal derruba uma recompensa durante o trajeto de retirada da mesma do aparato; registrado para cada lado separadamente. $\bigcirc$ lado não dominante tende a apresentar maior número de perdas.

\section{Análise de parâmetros no teste tabuleiro de Brinkman}

Os testes foram registrados em vídeo para a análise dos mesmos parâmetros anteriores de avaliação de preferência e dominância manuais. A fim de analisar - estabelecimento de padrões motores durante os períodos de teste no tabuleiro de Brinkman, realizou-se uma avaliação quantitativa da sequência espacial utilizada por cada animal para a retirada das recompensas comestíveis no tabuleiro. Para tal, foi utilizado um índice de sistematização da sequência motora, conforme anteriormente proposto ${ }^{27}$. Como parâmetro, utilizou-se uma sequência sistemática iniciando da extremidade esquerda do tabuleiro e finalizando na extremidade direita, conforme ilustrado na figura 3. Para o cálculo do índice, foram usados os valores numéricos correspondentes a uma sequência padrão e subtraídos dos valores numéricos resultantes da ordem crescente na sequência de retirada das recompensas realizada pelos animais. A sequência espectral, por exemplo, determinou valores elevados dos índices. A partir desses cálculos, foi analisada a variabilidade dos dados ao longo dos períodos de treinamento, a fim de detectar a presença de estabelecimento de hábito motor no teste por parte dos animais, que pôde ser observada quando da apresentação de baixa variabilidade entre as sessões consecutivas.

\section{Análises estatísticas}

Os dados obtidos foram organizados em planilha e submetidos a análises estatísticas utilizando os softwares BioEstat v5.0 e GraphPad Prism. Foram aplicados testes de normalidade para a detecção de extremos (Kolmogorov-Smirnov), seguidos de testes estatísticos para a determinação de preferência e/ou dominância manual, por meio da aplicação de teste t bicaudal para amostras pareadas, para comparação dos resultados individuais dos dados obtidos para cada lado nos testes. $O$ intervalo de confiança mínimo para a detecção de significância permaneceu em 95\% $(p<0,05)$.

\section{RESULTADOS}

\section{ANÁLISE DE PARÂMETROS DO TESTE STAIRCASE}

Os dados revelaram diferenças significativas entre os lados direito e esquerdo para seis dos sete animais experimentais nos seguintes parâmetros: latência de retirada da primeira recompensa, tempo para recuperação de todas as recompensas e uso de mão trocada. No parâmetro perda da recompensa, observou-se diferença significativa entre os desempenhos dos lados direito e esquerdo somente para um dos sete animais experimentais (Figura 4). Não foi observada diferença significativa nas comparações entre as médias obtidas nos modos valley e hill para qualquer dos parâmetros ou sujeitos analisados.

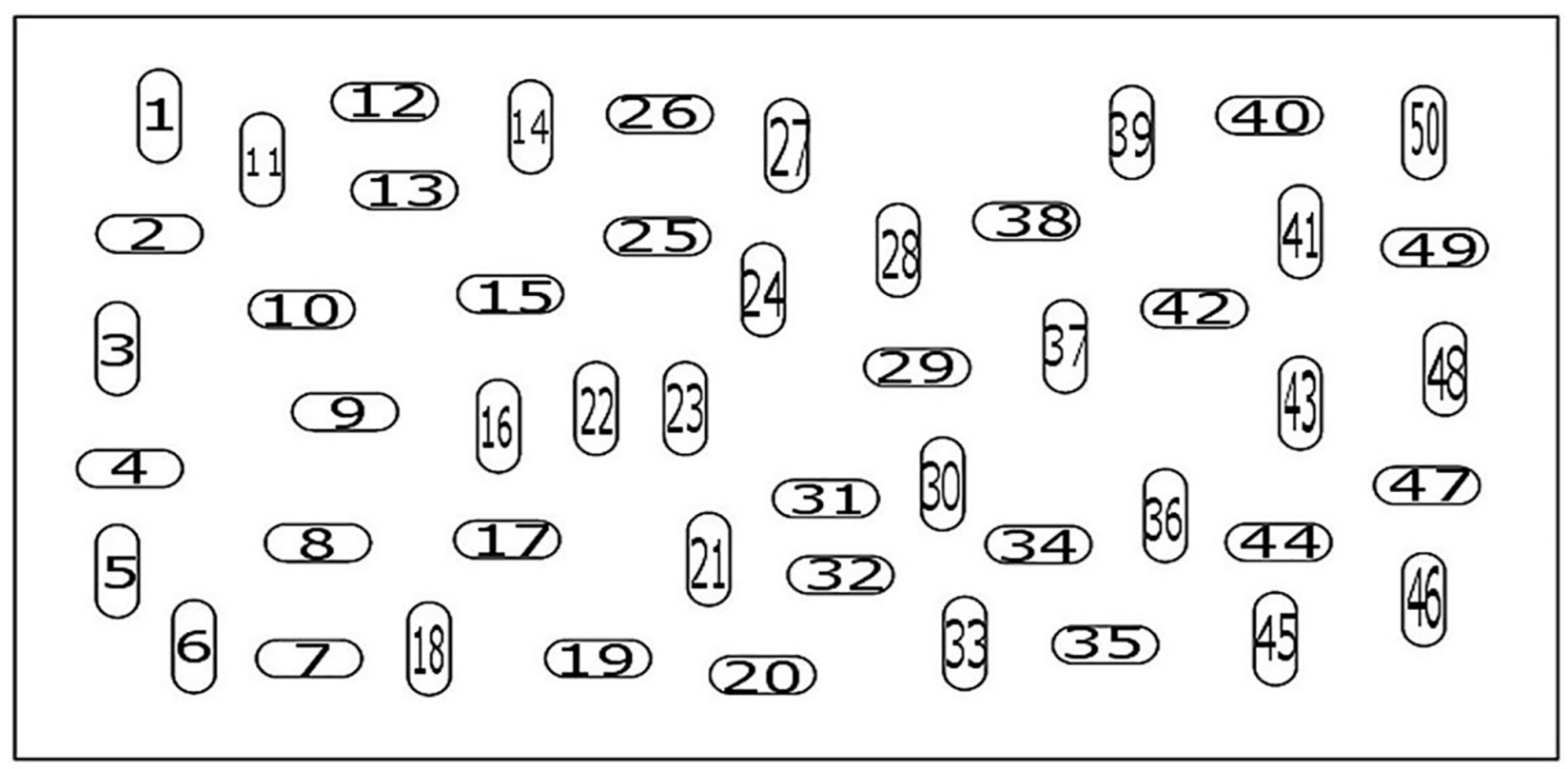

Figura 3 - Sequência padrão de análise para o cálculo do índice de sistematização da sequência espacial de retirada das recompensas pelos animais no tabuleiro de Brinkman 
LATÊNCIA DE RETIRADA DE PRIMEIRA RECOMPENSA

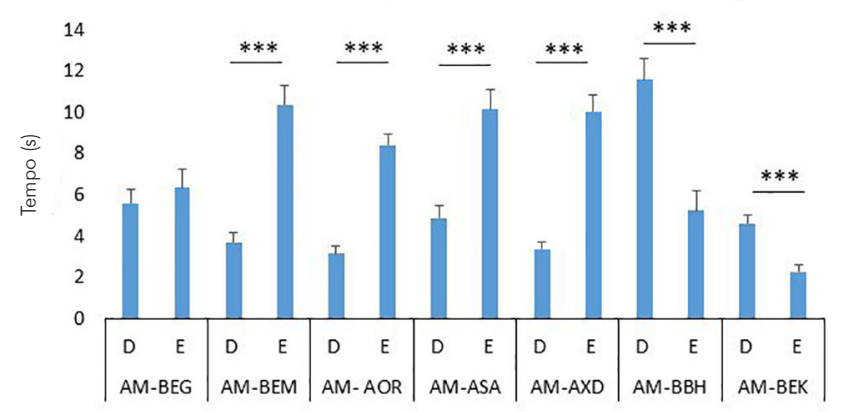

TEMPO PARA RECUPERAÇÃO DE TODAS AS RECOMPENSAS

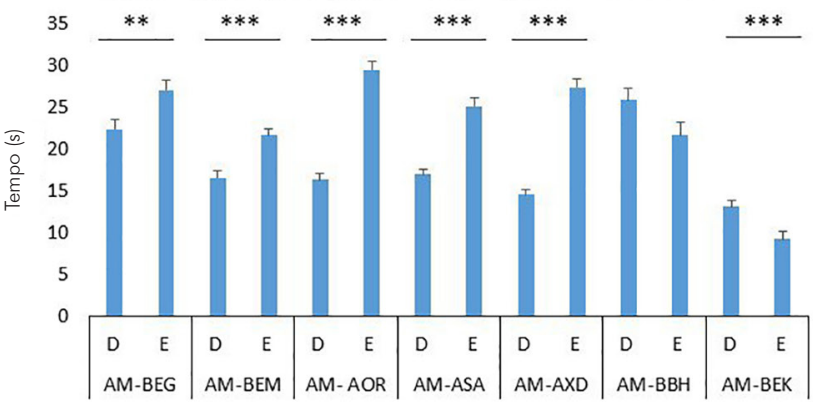

USO DE MÃO TROCADA

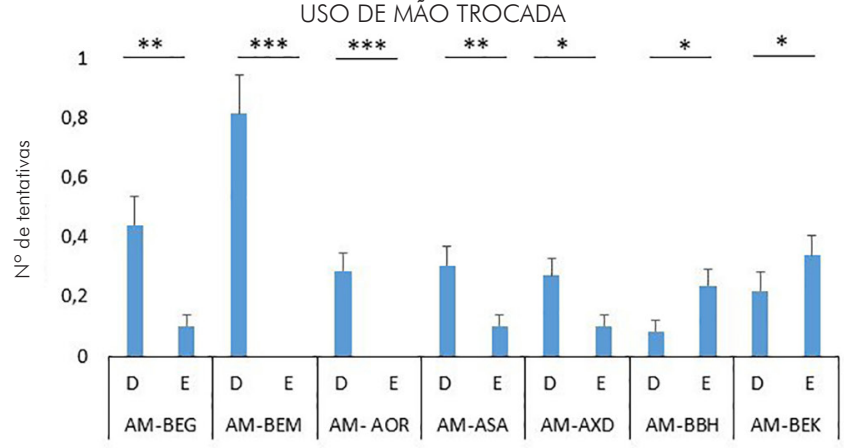

PERDA DE RECOMPENSA

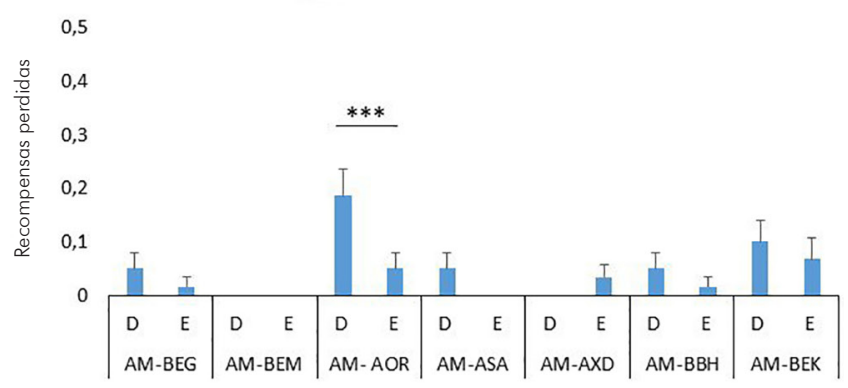

Teste $\mathrm{t},{ }^{* * *} \mathrm{p}<0,001$; $^{* *} \mathrm{p}<0,005 ;{ }^{*} \mathrm{p}<0,05$.

Figura 4 - Gráficos representativos das médias dos escores obtidos para as mãos direita e esquerda para cada animal no teste staircase segundo os parâmetros latência de retirada da primeira recompensa, uso de mão trocada, tempo para a recuperação de todas as recompensas e perda de recompensa

\section{ANÁLISE DE PARÂMETROS DO TABULEIRO DE BRINKMAN}

Para os quatro parâmetros analisados no tabuleiro de Brinkman, foram observadas diferenças significativas entre os desempenhos dos lados direito e esquerdo dos animais experimentais. Todavia, o número de sujeitos com resultados significativos para cada parâmetro foi inferior quando comparado aos resultados observados no teste staircase. Os dados estão ilustrados na figura 5 .

As análises das sequências espaciais utilizadas pelos animais para a recuperação das recompensas nos orifícios do tabuleiro de Brinkman revelaram redução da variabilidade das sequências ao longo das sessões comportamentais, com claro estabelecimento de padrão motor demonstrado pela redução do número de interseções nos trajetos estabelecidos pelos animais para cumprimento da tarefa no período (Figura 6).

ANÁLISE DA LATERALIDADE PELOS PARÂMETROS DE PREFERÊNCIA E DOMINÂNCIA OBSERVADOS NOS TESTES

O quadro 2 mostra os parâmetros que indicaram diferenças significativas de desempenho entre as mãos direita e esquerda de cada animal, o índice de significância observado e o lado que apresentou desempenho predominante. Observou-se que o teste staircase apresentou maior sensibilidade de detecção de atributos de preferência e dominância manual nos parâmetros latência de primeira recompensa, uso de mão trocada e tempo para recuperação de todas as recompensas, com significâncias observadas para a maior parte dos sujeitos experimentais. $O$ tabuleiro de Brinkman apresentou maior sensibilidade de detecção de dominância manual no parâmetro perda de recompensa. Alguns parâmetros apresentaram discrepância de lateralidade para preferência e dominância manuais dos animais entre os testes.

\section{DISCUSSÃO}

Neste estudo, observou-se os padrões de lateralização pela detecção da preferência e dominância manual em macacos S. apella, utilizando testes sensíveis à destreza manual. Foi investigada a hipótese de que os parâmetros observados nos testes staircase e tabuleiro de Brinkman podem ser instrumentos para predizer a preferência e/ou dominância manual nesses primatas. Os testes aplicados revelaram resultados consistentes que reforçam a distinção proposta anteriormente por Chatagny et al. ${ }^{6}$ acerca dos atributos de preferência e dominância manual em primatas.

Diferenças significativas de desempenho entre os lados direito e esquerdo foram observadas em ambos os testes, e alguns dos parâmetros apresentaram-se mais sensíveis à detecção de preferência ou dominância manual que outros. Ainda, os resultados sugerem que alguns animais possuem lateralidade mais acentuada que outros, com resultados significativos observados para a maior parte dos parâmetros. Nesses animais, os resultados indicativos de dominância e preferência manuais apresentaram maior concordância de lateralidade. Para a maior parte dos animais, houve melhores desempenhos para a mão direita, tanto para preferência quanto para dominância manual. 
LATÊNCIA DE RETIRADA DE PRIMEIRA RECOMPENSA

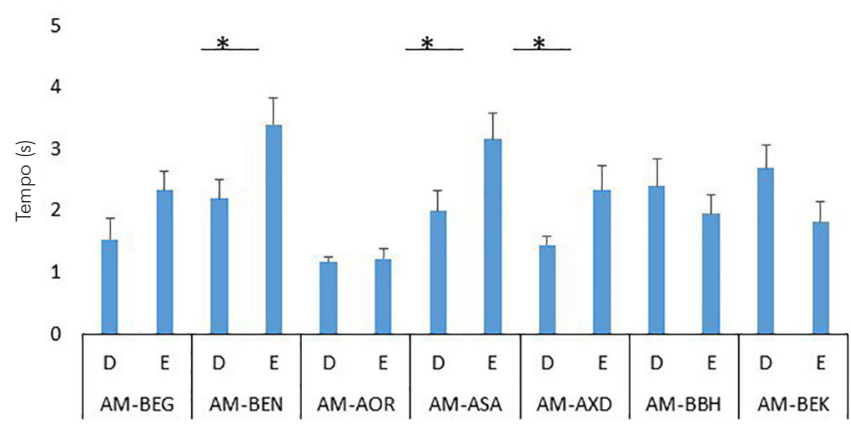

TEMPO PARA RECUPERAÇÃO DE TODAS AS RECOMPENSAS

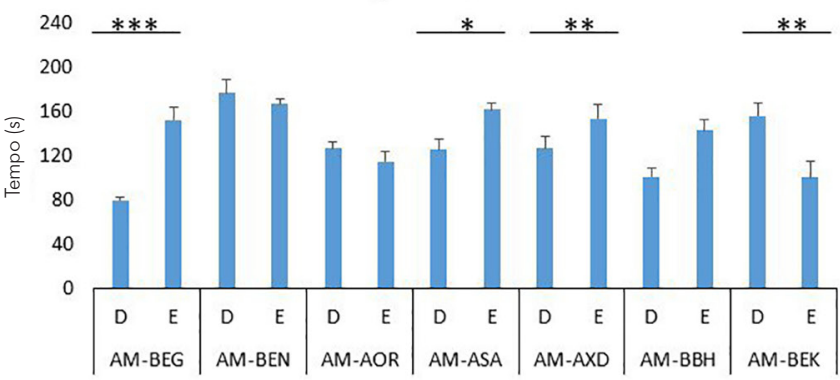

USO DE MÃO TROCADA

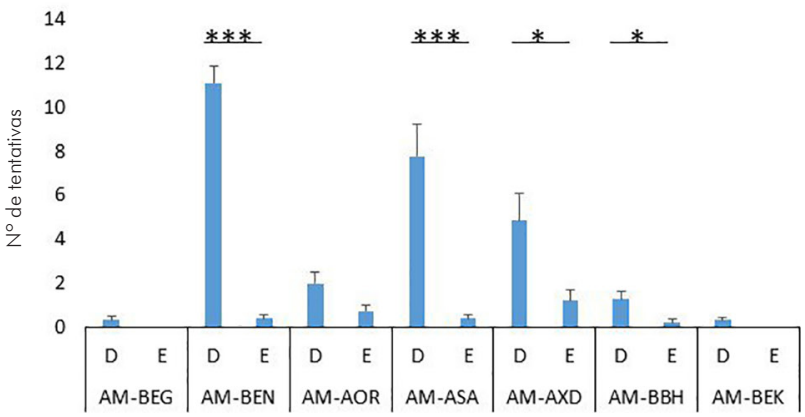

PERDA DE RECOMPENSA

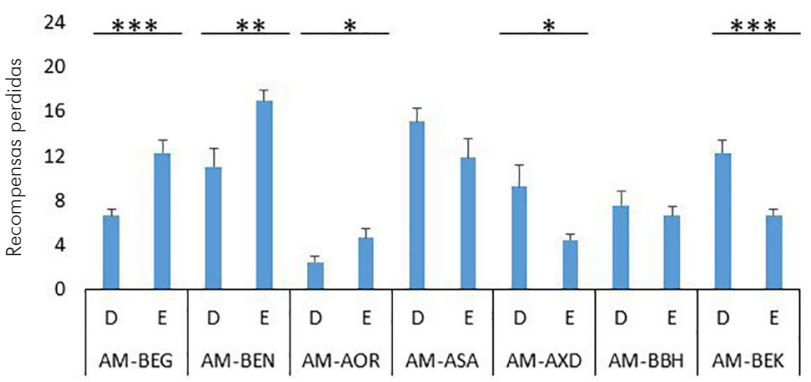

Teste $+{ }^{* * *} p<0,001 ;{ }^{* *} p<0,005,{ }^{*} p<0,05$.

Figura 5 - Gráficos representativos das médias dos escores obtidos para as mãos direita e esquerda para cada animal no tabuleiro de Brinkman segundo os parâmetros latência de retirada da primeira recompensa, uso de mão trocada, tempo para a recuperação de todas as recompensas e perda de recompensa

VARIABILIDADE DE SEQUÊNCIAS TEMPORAIS

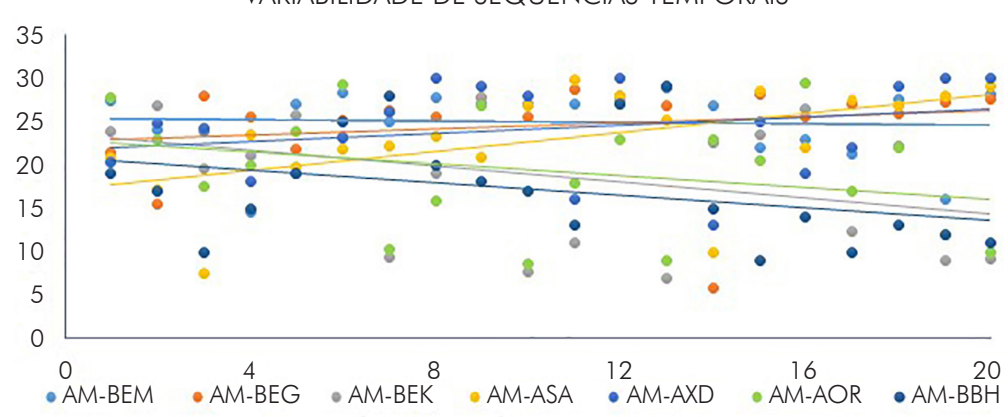

AM-BEM

17yns

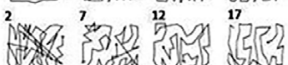

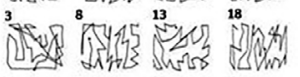

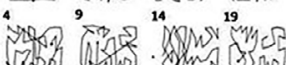

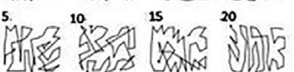

AM-BEK

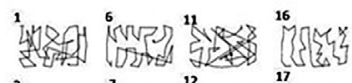

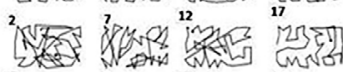

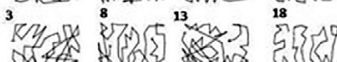

굴

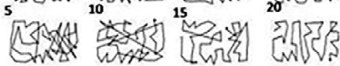

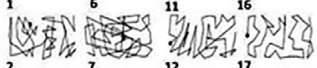

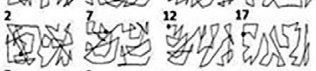

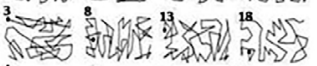

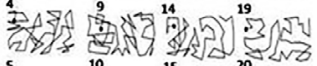

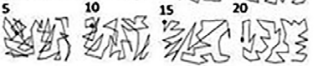

Gráfico: índices de sequência temporal obtidos nas sessões de teste para cada animal experimental. Esquemas: trajetórias executadas pelos animais para recuperação das recompensas nos orifícios distribuídos ao longo do tabuleiro de Brinkman, para cada uma das sessões de testes.

Figura 6 - Sequências espaciais utilizadas pelos animais experimentais para a recuperação das recompensas nos orifícios do tabuleiro de Brinkman 
Parâmetros

\begin{tabular}{|c|c|c|c|c|}
\hline \multirow[b]{2}{*}{ Animais experimentais } & \multicolumn{4}{|c|}{ Parâmetros } \\
\hline & $\begin{array}{l}\text { Latência de retirada de } \\
\text { primeira recompensa }\end{array}$ & Uso de mão trocada & $\begin{array}{l}\text { Tempo para a } \\
\text { recuperação de todas } \\
\text { as recompensas }\end{array}$ & Perda de recompensa \\
\hline \multicolumn{5}{|c|}{ Teste staircase } \\
\hline AM-BEG & $\mathrm{D}$ & $D^{* *}$ & $D^{* *}$ & $E$ \\
\hline AM-BEM & $\mathrm{D}^{* * *}$ & $\mathrm{D}^{* * *}$ & $D^{* * *}$ & - \\
\hline AM-AOR & $D^{* * *}$ & $\mathrm{D}^{* * *}$ & $\mathrm{D}^{* * *}$ & $\mathrm{E}^{* * *}$ \\
\hline AM-ASA & $D^{* * *}$ & $D^{* *}$ & $D^{* * *}$ & $E$ \\
\hline AM-AXD & $D^{* * *}$ & $D^{*}$ & $D^{* * *}$ & $D$ \\
\hline $\mathrm{AM}-\mathrm{BBH}$ & $\mathrm{E}^{* * *}$ & $E^{*}$ & E & $E$ \\
\hline AM-BEK & $\mathrm{E}^{* * *}$ & $E^{*}$ & $\mathrm{E}^{* * *}$ & $E$ \\
\hline \multicolumn{5}{|c|}{ Tabuleiro de Brinkman } \\
\hline AM-BEG & $\mathrm{D}$ & $\mathrm{D}$ & $D^{* * *}$ & $D^{* * *}$ \\
\hline AM-BEM & $D^{*}$ & $\mathrm{D}^{* * *}$ & $E$ & $D^{* *}$ \\
\hline AM-AOR & $\mathrm{D}$ & $\mathrm{D}$ & E & $D^{*}$ \\
\hline AM-ASA & $D^{*}$ & $D^{* * *}$ & $D^{*}$ & $E$ \\
\hline AM-AXD & $D^{*}$ & $D^{*}$ & $D^{* *}$ & $E^{*}$ \\
\hline $\mathrm{AM}-\mathrm{BBH}$ & $E$ & $D^{*}$ & $\mathrm{D}$ & $E$ \\
\hline AM-BEK & $E$ & $\mathrm{D}$ & $\mathrm{E}^{* *}$ & $E^{* * *}$ \\
\hline
\end{tabular}

O lado especificado $(\mathrm{D}=$ direita $\mathrm{E}=$ esquerda $)$ indica o melhor desempenho significativo para aquele determinado parâmetro. Teste $\mathrm{t}^{* * *} \mathrm{p}<0,001$; ${ }^{* *} p<0,005 ;{ }^{*} p<0,05$.

Quadro 2 - Lateralidade demonstrada pelos animais experimentais em cada parâmetro dos testes analisados

Os dados do presente estudo corroboram achados anteriores, que apontam para maior frequência de preferência pela mão direita tanto em primatas do gênero Cebus quanto de outros gêneros ${ }^{13-19,22,23,24}$. Ademais, a variação observada na distribuição dos atributos de preferência e dominância para os animais AM-AOR, AM-AXD e AM-BBH reforçam a hipótese de Chatagny et al. ${ }^{6}$ acerca da distinção entre os atributos de preferência e dominância manual e de sua não necessária correspondência entre os primatas. Todavia, se esses dados sugerem a presença de ambidestria ou a simples ocorrência de disparidade entre preferência e dominância, é uma questão que merece investigações mais profundas nas quais devem ser considerados os fatores a seguir.

Investigações anteriores demonstraram que resultados discrepantes com relação à preferência manual em primatas podem ser decorrentes da especificidade dos testes e parâmetros para a sua determinação. De um modo geral, a complexidade do teste empregado parece ser de grande relevância ${ }^{26,36}$. É importante considerar que a maior parte dos estudos investigando lateralidade em primatas não faz distinção entre os atributos de preferência e dominância manual, caracterizando apenas como preferência manual a apresentação de um desempenho superior de uma mão em relação à outra.
De um modo geral, testes que envolvam tarefas de grande habilidade são mais sensíveis à detecção da dominância manual, enquanto testes de baixa complexidade motora tendem a revelar mais claramente a preferência manual. $\bigcirc$ tabuleiro de Brinkman é largamente utilizado para estudos da determinação de lateralidade em primatas $37,38,39$, enquanto o teste staircase tem sido menos frequentemente descrito ${ }^{40}$. Ao mesmo tempo que o teste staircase permite uma avaliação conjunta da coordenação motora global e fina, o tabuleiro de Brinkman requer que o animal utilize a pegada em pinça, um dos movimentos de maior precisão, exclusivo dos primatas, que requer a cooperação coordenada de diversos músculos da mão e braço ${ }^{41}$. $\bigcirc$ teste staircase empregado neste estudo demonstrou maior sensibilidade para a detecção de lateralidade no parâmetro de preferência, enquanto o tabuleiro de Brinkman parecer ser mais sensível para a detenção de dominância manual, especialmente para o parâmetro perda da recompensa, o que pode ser resultante tanto da complexidade da tarefa de retirada (a partir de orifícios pequenos no tabuleiro de Brinkman comparado com sobre a superfície de um degrau no staircase) quanto do número de recompensas (50 no tabuleiro de Brinkman comparado a cinco no staircase) utilizados nas tarefas de retirada em cada um dos testes. Em conjunto, os testes demonstraram ser eficazes para a determinação da lateralidade em primatas. 
É importante considerar ainda que estes resultados podem sofrer influência do ganho de proficiência dos animais nos testes ao longo das sessões. As análises dos padrões motores no tabuleiro de Brinkman revelaram alterações no padrão de ordem de retirada das recompensas do tabuleiro ao longo do período de testes, com redução da variabilidade e criação de estratégias motoras por parte dos animais para a retirada das recompensas. Dados de estudos investigando lateralidade em primatas sugerem que macacos aprendem ou refinam a preferência manual pela experiência motora, sem diferenças observáveis no aprendizado pelos hemisférios ipsilateral e contralateral à mão de preferência ${ }^{6,27}$, diferentemente dos humanos, onde a dominância manual é uma característica congênita ${ }^{5}$. Dessa forma, é possível que as diferenças observadas aqui nos parâmetros de preferência e dominância manuais reflitam, pelo menos parcialmente, resultados de aprendizagem motora induzida pelas próprias sessões de testes. Se em um protocolo de maior duração essas diferenças oscilam ou estabilizam, é uma questão a ser investigada.

Análises anteriores do desempenho de macacos no tabuleiro de Brinkman demonstraram a existência de diferenças interindividuais consideráveis em estratégias e hábitos motores, que influenciam a precisão e os padrões de realização do teste em primatas ${ }^{27}$, semelhantes às diferenças de padrões motores individuais observadas em humanos desde a infância ${ }^{28}$. Padrões motores finos e complexos, como o tipo de pegada e estratégia motora (posição de dedos em relação ao tabuleiro), não foram analisados neste ensaio. No contexto da especificidade motora e dominância manual, forte influência é exercida pelo tamanho do objeto e pelo tipo de pegada sobre o desempenho no teste de Brinkman. Em humanos, por exemplo, um estudo focado em pegadas de precisão identificou pelo menos sete tipos de pegada envolvendo - pólex e diversas combinações e posturas assumidas em relação aos outros dígitos ${ }^{42}$. Como neste estudo não foi realizada uma análise detalhada desses padrões, não se pode afirmar em que extensão as diferenças individuais de padrão de pegada entre os macacos podem ter influenciado a variabilidade dos dados aqui descritos.

\section{LIMITAÇÕES TÉCNICAS}

O presente estudo apresenta limitações que devem ser consideradas quando da interpretação de seus resultados. Limitações inerentes a estudos com primatas incluem preferências individuais dos animais por determinado tipo de recompensa ou a forma e tamanho na qual as mesmas se apresentam, que podem ser fontes de variação nos resultados dos testes. Ainda, - rapport entre cuidador e primata pode afetar os resultados dos testes de acordo com o pesquisador que o está aplicando ${ }^{43}$. A fim de minimizar tais efeitos, as recompensas utilizadas eram trocadas diariamente, e aquelas utilizadas para teste em um determinado dia eram as mesmas para todos os animais. Análises de normalidade foram aplicadas para a exclusão de dados extremos da amostra. Todos os testes foram aplicados pelos mesmos dois pesquisadores, que diariamente ministravam em conjunto a tarefa. Entretanto, o número reduzido de animais experimentais e o curto período de análise dos testes podem ter limitado a observação de diferenças estatisticamente significativas devido ao baixo poder da amostra. As comparações dos dados aqui obtidos com estudos anteriores é de certa forma limitada, uma vez que a lateralidade e a destreza manuais variam entre as diferentes espécies de primatas ${ }^{16,18,27}$, e suas determinações são influenciadas por diferenças nos protocolos de aplicação e tipos de teste utilizados ${ }^{44}$.

\section{CONCLUSÃO}

As relações observadas neste estudo, consideradas as limitações técnicas, adicionam evidências a favor dos conceitos e da separação entre os fenômenos de preferência e dominância manual anteriormente propostos. Os testes staircase e tabuleiro de Brinkman apresentaram diferentes sensibilidades na detecção da preferência e da dominância manuais, e sua utilização deve ser cuidadosamente planejada e analisada em estudos dedicados à avaliação da lateralidade em primatas. Os macacos S. apella, assim como observado em outros estudos e com outras espécies, apresentaram predominante lateralização direita, especialmente no parâmetro de preferência manual, não havendo necessariamente estreita correspondência entre preferência e dominância manuais. A análise para determinação da lateralidade em termos de preferência e dominância, apesar de sua limitada descrição na literatura, é fator essencial para estudos envolvendo diversas condições motoras e deve ser adotada como guia para decisões e avaliação de resultados experimentais.

\section{AGRADECIMENTOS}

A Obadias dos Reis Silva e à Sheila Tetsume Makiama, pelo auxílio no manejo e treinamento dos animais experimentais.

\section{APOIO FINANCEIRO}

O presente estudo recebeu financiamento do Programa de Investigação e Inovação Horizonte 2020 da União Europeia, sob o contrato n. ${ }^{\circ} 767092$.

\section{CONFLITOS DE INTERESSES}

Os autores declaram que não há conflitos de interesses.

\section{CONTRIBUIÇÃO DOS AUTORES}

Todos os autores contribuíram para a idealização do estudo, análise e/ou interpretação dos dados e escrita deste manuscrito, aprovando sua publicação. Estes se declaram responsáveis pelo conteúdo, garantindo sua acurácia e integridade. 


\section{REFERÊNCIAS}

1 Courtine G, Bunge MB, Fawcett JW, Grossman $R G$, Kaas $J H$, Lemon $R$, et al. Can experiments in nonhuman primates expedite the translation of treatments for spinal cord injury in humans? Nat Med. 2007 May;13(5):561-6.

2 Alstermark B, Pettersson LG, Nishimura $Y$, Yoshino-Saito K, Tsuboi F, Takahashi M, et al. Motor command for precision grip in the macaque monkey can be mediated by spinal interneurons. J Neurophysiol. 2011 Jul;106(1):122-6.

3 Kinoshita M, Matsui R, Kato S, Hasegawa T, Kasahara H, Isa K, et al. Genetic dissection of the circuit for hand dexterity in primates. Nature. 2012 Jul;487(7406):235-8.

4 Zhao D, Hopkins WD, Li B. Handedness in nature: first evidence on manual laterality on bimanual coordinated tube task in wild primates. Am J Phys Anthropol. 2012 May;1 48(1):36-44.

5 Warren JM. Handedness and laterality in humans and other animals. Physiol Psychol. 1980;8(3):351-9.

6 Chatagny P, Badoud S, Kaeser M, Gindrat AD, Savidan J, Fregosi $M$, et al. Distinction between hand dominance and hand preference in primates: a behavioral investigation of manual dexterity in nonhuman primates (macaques) and human subjects. Brain Behav. 2013 Sep;3(5):575-95.

7 Serrien DJ, Ivry RB, Swinnen SP. Dynamics of hemispheric specialization and integration in the context of motor control. Nat Rev Neurosci. 2006 Feb;7(2):160-6.

8 Gotts SJ, Jo HJ, Wallace GL, Saad ZS, Cox RW, Martin A. Two distinct forms of functional lateralization in the human brain. Proc Natl Acad Sci USA. 2013 Sep;110(36):E3435-44.

9 Hepper PG, Shahidullah S, White R. Handedness in the human fetus. Neuropsychologia. 1991;29(1 1):1107-11.

10 Raymond M, Pontier D. Is there geographical variation in human handedness? Laterality. 2004 Jan;9(1):35-51.

11 Papademetriou E, Sheu CF, Michel GF. A meta-analysis of primate hand preferences, particularly for reaching. J Comp Psychol. 2005 Feb;1 19(1):33-48.

12 Murata Y, Higo N, Oishi T, Yamashita A, Matsuda $K$, Hayashi M, et al. Effects of motor training on the recovery of manual dexterity after primary motor cortex lesion in macaque monkeys. J Neurophysiol. 2008 Feb;99(2):773-86.

13 Masataka N. Population-level asymmetry of hand preference in lemurs. Behaviour. 1989 Jan;1 10(1-4):244-7.
14 Ward JP, Milliken GW, Dodson DL, Stafford DK, Wallace M. Handedness as a function of sex and age in a large population of Lemur. J Comp Psychol. 1990 Jun;104(2):167-73.

15 Ward JP, Milliken GW, Stafford DK. Patterns of lateralized behavior in prosimians. In: Ward J, Hopkins WD, editors. Primate laterality. New York: Springer; 1993. p. 43-74.

16 Fagot J, Vauclair J. Manual laterality in nonhuman primates: a distinction between handedness and manual specialization. Psychol Bull. 1991 Jan;109(1):76-89.

17 Spinozzi G, Castorina MG, Truppa V. Hand preferences in unimanual and coordinated-bimanual tasks by tufted capuchin monkeys (Cebus apella). J Comp Psychol. 1998; $112(2): 183-91$.

18 Lacreuse A, Parr LA, Smith HM, Hopkins WD. Hand preferences for a haptic task in chimpanzees (Pan troglodytes). Int J Primatol. 1999 Dec; 20(6):867-81.

19 Hopkins WD, Phillips KA, Bania A, Calcutt SE, Gardner M, Russell J, et al. Hand preferences for coordinated bimanual actions in 777 great apes: implications for the evolution of handedness in hominins. J Hum Evol. 2011 May;60(5):605-11.

20 Silva Jr JS. Especiação nos macacos-prego e caiararas, gênero Cebus Erxleben, 1777 (Primates, Cebidae) [tese]. Rio Janeiro (RJ): Universidade Federal do Rio de Janeiro; 2001.

21 Alfaro JWL, Silva Jr JS, Rylands AB. How different are robust and gracile capuchin monkeys? An argument for the use of Sapajus and Cebus. Am J Primatol. 2012 Apr;74(4):273-86.

22 Masataka N. Handedness of capuchin monkeys. Folia Primatol. 1990;55(3-4):189-92.

23 Westergaard GC, Byrne G, Suomi SJ. Handedness and cortisol in tufted capuchin monkey infants. Dev Psychobiol. 2000 Apr;36(3):213-7.

24 Westergaard GC, Suomi SJ. Hand preference for stone artefact production and tool-use by monkeys: possible implications for the evolution of right-handedness in hominids. J Hum Evol. 1996 Apr;30(4):291-8.

25 Phillips KA, Sherwood CC. Primary motor cortex asymmetry is correlated with handedness in capuchin monkeys (Cebus apella). Behav Neurosci. 2005;119(6):1701-4.

26 Spinozzi G, Laganà T, Truppa V. Hand use by tuffed capuchins (Cebus apella) to extract a small food item from a tube: digit movements, hand preference, and performance. Am J Primatol. 2007 Mar;69(3):336-52. 
27 Kaeser M, Chatagny P, Gindrat AD, Savidan J, Badoud S, Fregosi $M$, et al. Variability of manual dexterity performance in non-human primates (Macaca fascicularis). Int J Comp Psychol. $2014 ; 27(2): 295-325$.

28 Kakebeeke TH, Caflisch J, Chaouch A, Rousson V, Largo RH, Jenni OG. Neuromotor development in children. Part 3: motor performance in 3- to 5-year-olds. Dev Med Child Neurol. 2013 Mar;55(3):248-56.

29 Bashir S, Kaeser M, Wyss A, Hamadjida A, Liu Y, Bloch J, et al. Short-term effects of unilateral lesion of the primary motor cortex (M1) on ipsilesional hand dexterity in adult macaque monkeys. Brain Struct Funct. 2012 Jan;217(1):63-79.

30 Eslamboli A, Baker HF, Ridley RM, Annett LE. Sensorimotor deficits in a unilateral intrastriatal 6-OHDA partial lesion model of Parkinson's disease in marmoset monkeys. Exp Neurol. 2003 Oct; 183(2):418-29.

31 Eslamboli A, Cummings RM, Ridley RM, Baker HF, Muzyczka N, Burger C, et al. Recombinant adeno-associated viral vector (rAAV) delivery of GDNF provides protection against 6-OHDA lesion in the common marmoset monkey (Callithrix jacchus). Exp Neurol. 2003 Nov; 184(1):536-48.

32 Hamadjida A, Wyss AF, Mir A, Schwab ME, Belhai-Saif A, Roviller EM. Influence of anti-Nogo-A antibody treatment on the reorganization of callosal connectivity of the premotor cortical areas following unilateral lesion of primary motor cortex (M1) in adult macaque monkeys. Exp Brain Res. 2012 Nov;223(3):321-40.

33 Marshall JWB, Ridley RM. Assessment of cognitive and motor deficits in a marmoset model of stroke. ILAR J. 2003;44(2): 153-60.

34 Baird AL, Meldrum A, Dunnett SB. The staircase test of skilled reaching in mice. Brain Res Bull. 2001 Jan;54(2):243-50.

35 Schmidlin E, Kaeser M, Gindrat AD, Savidan J, Chatagny P, Badoud S, et al. Behavioral assessment of manual dexterity in non-human primates. J Vis Exp. 2011 Nov;(57):3258.
36 Uomini NT. The prehistory of handedness: archaeological data and comparative ethology. J Hum Evol. 2009 Oct;57(4):411-9.

37 Liu Y, Rouiller EM. Mechanisms of recovery of dexterity following unilateral lesion of the sensorimotor cortex in adult monkeys. Exp Brain Res. 1999 Sep;128(1-2):149-59.

38 Kaeser M, Wyss AF, Bashir S, Hamadjida A, Liu $Y$, Bloch J, et al. Effects of unilateral motor cortex lesion on Ipsilesional hand's reach and grasp performance in monkeys: relationship with recovery in the contralesional hand. J Neurophysiol. 2010 Mar;103(3): 1630-45.

39 Freund P, Schmidlin E, Wannier T, Bloch J, Mir A, Schwab ME, et al. Antibody treatment promotes recovery of manual dexterity after unilateral cervical lesion in adult primates- -re-examination and extension of behavioral data. Eur J Neurosci. 2009 Mar;29(5):983-96.

40 Santisakultarm TP, Kersbergen CJ, Bandy DK, Ide DC, Choi SH, Silva AC. Two-photon imaging of cerebral hemodynamics and neural activity in awake and anesthetized marmosets. J Neurosci Methods. 2016 Sep;271:55-64.

41 Porter R. Corticospinal influences on the spinal cord machinery for movement. In: Porter R, Lemon R. Corticospinal function and voluntary movement. New York: Oxford University Press; 1993. p. 122-209. (Monographs of the Physiological Society; no. 45$)$

42 Wong YJ, Whishaw IQ. Precision grasps of children and young and old adults: individual differences in digit contact strategy, purchase pattern, and digit posture. Behav Brain Res. 2004 Sep; 154(1):113-23.

43 Waitt C, Buchanan-Smith HM, Morris K. The effects of caretaker-primate relationships on primates in the laboratory. J Appl Anim Welf Sci. 2002;5(4):309-19.

44 Marteniuk RG. Individual differences in motor performance and learning. Exerc Sport Sci Rev. 1974 Jan;2(1):103-30. 